\title{
Simulation-based acceptance testing for unmanned ground vehicles
}

\author{
Amir Sadrpour* \\ Department of Industrial and Operations Engineering, \\ The University of Michigan, \\ 1205 Beal Ave., Ann Arbor, \\ MI 48109-2117, USA \\ E-mail: sadrpour@umich.edu \\ ${ }^{*}$ Corresponding author

\section{Jionghua Jin}

Department of Industrial and Operations Engineering,

The University of Michigan,

2855 IOE Building, 1205 Beal Avenue,

Ann Arbor, MI 48109-2117, USA

E-mail: jhjin@umich.edu

\section{A. Galip Ulsoy}

Department of Mechanical Engineering,

The University of Michigan,

2266 G.G. Brown Building, 2350 Hayward Street,

Ann Arbor, MI 48109-2125, USA

Fax: (734)647-3170

E-mail: ulsoy@umich.edu

\section{Hyo Jong Lee}

Department of Mechanical Engineering,

MS in Mechanical Engineering from University of Michigan,

107-1401 SongnimMaeul Apt.,

Hagi-dong, Yuseong-gu,

Daejeon 305-759, South Korea

E-mail: loveara@gmail.com

\begin{abstract}
Performing physical acceptance tests of an Unmanned Ground Vehicle (UGV) can be expensive and time-consuming. This paper discusses simulation-based acceptance testing and failure analysis for UGVs. Both dynamic and static simulation models are developed. A systematic statistical testing approach is presented to quantitatively assess when a simple static simulation model can be used to approximate a complex dynamic simulation. Results show that a static simulation can be used to determine the required joint
\end{abstract}


motor torques under slow operation speeds. It also shows that a dynamic simulation model is needed to determine the maximum allowable moving speeds for UGVs to safely operate on roads with various levels of roughness and bumpiness.

Keywords: acceptance testing; UGV; unmanned ground vehicle; dynamic simulation; statistical hypothesis test.

Reference to this paper should be made as follows: Sadrpour, A., Jin, J., Ulsoy, A.G. and Lee, H.J. (2013) 'Simulation-based acceptance testing for unmanned ground vehicles', Int. J. Vehicle Autonomous Systems, Vol. 11, No. 1, pp.62-85.

Biographical notes: Amir Sadrpour is a third year PhD Student in the Department of Industrial and Operations Research Engineering at the University of Michigan. He received his MS Degree from the University of Michigan (2010), and his BS Degree from Ohio State University (2008). Joining the Ground Robotic Reliability Center (GRRC) at the University of Michigan in 2010, the focus of his research has been on Unmanned Ground Vehicles (UGVs) reliability modelling, prediction, and acceptance testing. His primary research interests include simulation-based acceptance testing and system reliability assessment with limited failure data in the presence of environmental uncertainty.

Jionghua (Judy) Jin is Professor of Industrial and Operations Engineering at the University of Michigan. She received her $\mathrm{PhD}$ Degree from the University of Michigan in 1999. Her research focuses on data fusion for complex system improvement with the goal of developing novel methodologies for variation reduction, condition monitoring and fault diagnosis, process control, knowledge discovery and decision making. She has received a number of awards including the NSF CAREER Award in 2002 and the PECASE Award in 2004, six Best Paper Awards during 2005 2009. She is a member of ASME, ASQC, IEEE, IIE, INFORMS, and SME.

A. Galip Ulsoy is the C.D. Mote, Jr. Distinguished University Professor of Mechanical Engineering and the William Clay Ford Professor of Manufacturing at University of Michigan, Ann Arbor. He received the $\mathrm{PhD}$ from University of California at Berkeley (1979), the MS Degree from Cornell University (1975), and the BS Degree from Swarthmore College (1973). His research interests are in the dynamics and control of mechanical systems. $\mathrm{He}$ has received numerous awards, including 2008 Rufus T. Oldenburger Medal from ASME. He is a member of the National Academy of Engineering and is a Fellow of ASME, SME and IFAC.

Hyo Jong Lee is a former student of the University of Michigan's Ground Robotics Reliability Center (GRRC). He received his Bachelor's and Master's Degrees in mechanical engineering from the University of Michigan, Ann Arbor in 2008 and 2010 respectively. From September 2008 to December 2009, he held a Graduate Student Research Assistant (GSRA) position with the University of Michigan's GRRC. During this time, his research mainly focused on developing methods to improve reliability of UGV systems. His primary research interests include robotic systems and control, mechatronics system design, developing methods for UGV reliability improvement, and UGV acceptance testing. 


\section{Introduction}

In 2004, the US Army was using about 160 robots in Iraq and Afghanistan (Purdy, 2007). This number grew to approximately 4000 in 2007 and continued to climb to about 8000 in 2010. With the rapid increase of Unmanned Ground Vehicle (UGV) usage in military operations, one primary concern of robotics researchers and users is UGV reliability (Tilbury and Ulsoy, 2011). Studies of mobile robots used in Urban Search and Rescue (US\&R) and Military Operations in Urban Terrain (MOUT) have shown a Mean Time Between Failures (MTBF) in the field of 6-20 h, well below the desired $96 \mathrm{~h}$. established by the Test and Evaluation Coordination Office (TECO), part of the Maneuver Support Center at Ft. Leonard Wood (Kramer and Murphy, 2006). Some of the failures are due to manufacturing defects or subtle interactions between components, and these failures could be detected and prevented prior to field deployment. However, other failures are due to uncertain operating environments, misuse by operators, and insufficient understanding of failure modes. Therefore, it is important to develop an acceptance test to provide better understanding of the failure modes and to ensure that such systems meet their reliability goals. Although such testing methods are widely used in various engineering applications, there is still no general guidance for UGV acceptance tests in terms of system reliability. Thus, the purpose of this paper is to suggest research ideas that might provide a basis for the development of an acceptance test for small UGVs or mobile robots.

Some of the earliest works on UGV reliability and acceptance testing were done by Murphy et al. (Carlson et al., 2004; Carlson and Murphy, 2003, 2005; Carlson, 2004; Kramer and Murphy, 2006; Micire, 2002). Murphy discussed a methodology similar to a final factory acceptance test, i.e. a test usually performed by a manufacturer prior to shipping. In contrast, this paper discusses a methodology for simulation-based acceptance testing in which simulations are used to identify the safe operation boundaries or the worst-case scenarios in UGV operations.

The proposed simulation approach, which is considered a complementary tool for improving physical acceptance tests, has the following advantages that overcome some of the limitations of the physical tests:

- The simulation approach is time- and cost-effective, since it does not require expensive physical test facilities, expert operators to work with the UGV during the test, or various test apparatuses.

- Simulation testing can be conducted on the full operational range of the UGV, beyond the limitations that the test facilities and apparatus may offer.

- Simulation results can be used to develop the boundaries of safe operation for UGVs for various scenarios. These boundaries can be potentially used for

(i) improving UGV design to prevent UGVs from functional failures due to design deficiencies

(ii) developing an intelligent human-UGV interface to avoid human operational failures

(iii) providing a systematic guideline in the design of the essential physical testing scenarios for reducing physical test time and cost. 
- It can help identify an optimal design or operational scheme, which exists beyond the currently available UGV products.

In this paper, two sets of commonly encountered UGV failure scenarios are investigated: the first set studies joint torque saturation and rollover when the UGV attempts to lift various loads; and the second set focuses on the suspension system and flip over failures when the vehicle is operated on bumpy terrains with mild roughness. To determine the UGV's safe operating range, we examined both dynamic and static simulation models for a wheeled ground vehicle whose size and weight is within the typical range of a small mobile robot.

The paper is organised as follows. Section 2 discusses related works in UGV reliability and acceptance testing. Section 3 presents our simulation-based acceptance testing methodology. Section 4 studies two failure modes associated with UGV operations: the joint saturation of the robot actuators and the rollover failure. To perform the study, we conduct both static and dynamic simulations and compare their performances using statistical hypothesis testing. Section 5 presents a similar analysis concerning two other failure modes: excessive mechanical shocks and vehicle flip over. Finally, the concluding remarks are outlined in the final section.

\section{Acceptance testing past and present}

This paper is intended to help users of UGVs gain a better understanding of the performance and reliability of a vehicle in various operational environments. Therefore, it is important to understand failure modes associated with current UGV systems. In Carlson et al. (2004), a novel taxonomy of UGV failures is introduced, which classifies failures into two major categories: physical failures and human operational failures. The physical failures are further studied based on the most common subsystems used in UGV platforms: effector, sensor, control system, power, and communications. Human operational failures are subdivided into mis-design specifications and human-robot interaction.

Previous work by the Center for Robot Assisted Search and Rescue (CRASAR) includes 13 studies and 15 different models of field robots in US\&R or military operations (Carlson and Murphy, 2003; Carlson, 2004). This study showed an overall MTBF of $8 \mathrm{~h}$ and an availability of less than $50 \%$. The effectors were the most common cause of failures (39\% of overall failures) and the control systems were next, with $29 \%$.

To ensure that field robots meet performance requirements and reliability targets, it is important to develop acceptance and performance testing standards for UGVs. Some preliminary work on UGV acceptance testing has been done by Murphy et al. In Kramer and Murphy (2006), the role of endurance testing for rescue and safety robots is discussed. It describes a methodology for endurance testing recommended for a certain class of robots. A six-hour endurance test was developed for a commercially available rescue robot. The test uncovered failures under certain conditions and the sources of the failures. In addition, the test data identified key design and manufacturing issues. Endurance and agility tests were also conducted at the US Army Aberdeen Test Center (ATC) on small size UGVs. The robots were tested under a variety of operating conditions to ensure their reliability in the field (Tricomo, 2009). 
In terms of performance standards, the Department of Homeland Security (DHS) initiated an effort in 2004 to develop performance standards for US\&R robots (Messina and Jacoff, 2006). To ensure that applicable technologies are relatively easy to use and integrate efficiently into existing systems, standardised test methods were needed. Therefore, the DHS Science and Technology Directorate initiated an effort in 2004 with National Institute of Standards and Technology (NIST) to develop comprehensive standards to support development, testing, and certification of effective robotic technologies for US\&R applications. These standards address robot mobility, sensing, navigation, and human system interaction. Recently, Pepper et al. (2007) used the standardised physical tests in NIST to validate the UGV's simulation models under given scenarios.

There are major limitations that almost all of the above acceptance and performance testing methods share. Due to limited testing facilities and apparatus, often only a small range of operational requirements can be physically tested. Additionally, conducting physical tests can be time consuming and tedious. Moreover, there are many uncontrollable or hard-to-control factors in physical tests, such as variations in different operators' knowledge and operating skills, environmental condition changes, etc., which may lead to inevitable variations in the physical testing results.

In contrast to the physical tests, the simulation-based tests can be very time efficient, since they can be fully automated and a variety of scenarios can be easily integrated into the simulation. Moreover, simulation-based evaluation methods have played an increasing role in complementing physical tests in other industries, such as aerospace and automotive (Norris, 1995; Guonian et al., 2010). In summary, the advantages that simulation-based acceptance testing offers makes it a complementary tool for physical acceptance testing. Therefore, this paper investigates simulation-based acceptance testing of UGVs to provide a faster and easier method to develop performance testing and to determine robot reliability.

\section{Proposed methodology}

Acceptance tests play an important role in the verification and demonstration of key performance requirements and system reliability of UGVs. To establish an acceptance test, the essential performance requirements and efficient test scenarios for each of the performance requirements need to be determined. These test scenarios emulate UGV operations and user environments, and ensure that the system meets the performance requirements and reliability goals.

In this paper, we propose a methodology for simulation-based acceptance testing which provides an efficient complement to physical acceptance testing (see Figure 1). To conduct the study, static and dynamic simulations for a few typical acceptance testing scenarios are developed. The settings represent tasks that are conventional in UGV operations and missions. These test plans include several UGV failure scenarios consisting of joint torque saturation, rollover, suspension system failure and flip over failures.

Our simulation-based acceptance testing is based on two models: dynamic and static simulation models. Each simulation model offers advantages and disadvantages, and Table 1 provides a qualitative summary of the key differences between them. Dynamic simulation can include a variety of environmental conditions which makes its results 
more accurate, whereas static simulation is more straightforward and quick to construct. Using the static simulation, we can readily derive the boundaries of safe operation through closed form static equations, while, in the dynamic case, these boundaries are obtained by exploring the large space of dynamic parameters and environments through simulations.

Figure 1 Proposed methodology for simulation-based acceptance testing and development of boundaries of safe operation

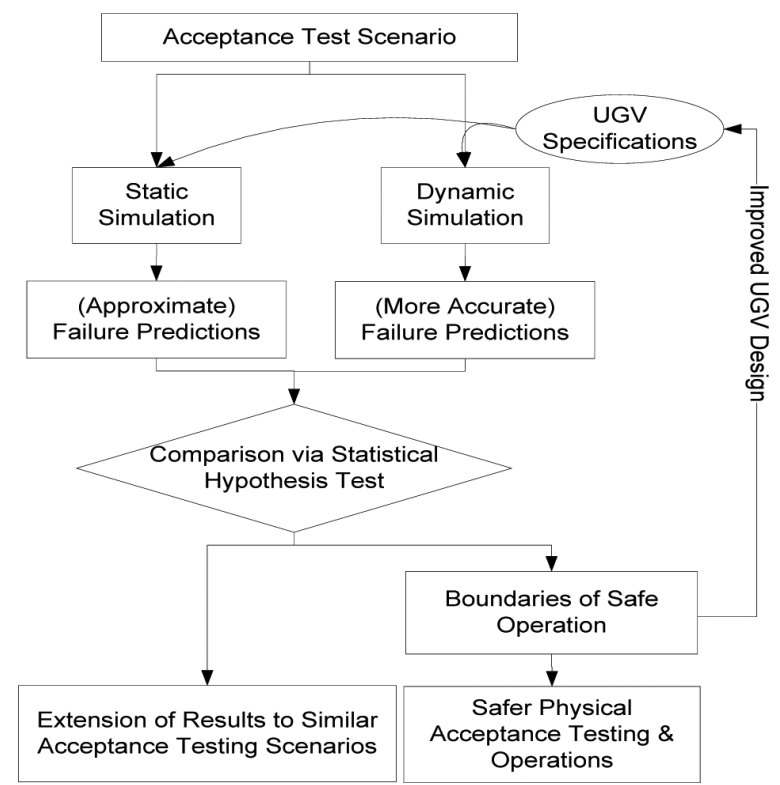

Table 1 Comparison of dynamic and static simulations

\begin{tabular}{lll}
\hline Characteristic & Dynamic simulation & Static simulation \\
\hline Complexity & Complex & Straight forward \\
Time/cost & Time consuming & Quick \\
Accuracy & Accurate & Approximate \\
Failure boundary identification & Difficult & Quick \\
\hline
\end{tabular}

Static simulation is more time efficient, but may not always be an acceptable approximation for dynamic simulation when the failure mechanism in a scenario is significantly affected by the dynamic characteristics of the UGV. Therefore, there is a need to develop a systematic method to quantitatively ensure when a static analysis is satisfactory. For this purpose, dynamic and static simulation results are compared by proposing a statistical hypothesis testing, which is used to judge whether both simulations will make a consistent decision on the failure state for a given scenario. The purpose of using a statistical hypothesis test is to consider inherent performance variation due to operational or environmental uncertainties. As will be discussed in the later sections, the outcome of the statistical hypothesis test, as to when static simulations can be used to approximate more complex dynamic simulations for a given scenario, can be extended to similar acceptance testing scenarios that share the same fundamental characteristics. 
The simulation results can help further identify the boundaries of safe operation, which can be used as a guideline to design efficient physical test scenarios and prevent expensive damages to a UGV during tests. Moreover, from the design perspective, the margin between the operational state and the design specification can indicate the failure probability or risk for conducting a given mission task. This will also provide us with a better understanding of failure mechanism, and guide us to improve the design of UGVs.

\section{Study of torque saturation and rollover failures}

\subsection{Scenario description}

In the first scenario, the robot lifts and moves an object with a known mass using its manipulator arm. The possible failure modes associated with this scenario are the inability of the arm actuators to provide the necessary torque for lifting the load, and also UGV rollover during the same lifting operation.

While it is desirable that the arm movements occur at a constant angular velocity, in reality speed variations occur, specially at low angular velocities arising from a variety of reasons such as variations in the power source, manufacturing limitations, interaction among components in the system, etc. Several papers have discussed the presence of these factors and methods to reduce the negative effect of speed and torque ripple in robot actuators by utilising effective control systems (Ren et al., 2009; Lam et al., 2000; Godler et al., 1994). Nonetheless, even with the use of feedback control, speed variations are not entirely eliminated. Moreover, since this scenario primarily focuses on actuator torque saturation, and speed and torque ripples influence this measure, their effect is considered in the dynamic simulation.

Electric motors are typically used in robot actuators. The accuracy of these motors mainly depends on the applications. For the purpose of this study, we assume that the actuator is using an effective control system to eliminate major harmonic trends in the ripples. With the major trends removed, the remaining disturbances are assumed to be random. Therefore, the speed ripples are modelled using white noise. The magnitude of the ripples are considered to be around $1 \%$ of the maximum operating speed of the robot arm, i.e. $0.3-0.5 \mathrm{deg} / \mathrm{sec}$ for a maximum speed of about $30-50 \mathrm{deg} / \mathrm{sec}$.

\subsection{Study of torque saturation failure}

\subsubsection{Static simulation framework}

Consider a system consisting of a wheeled platform and a two-link planar robot arm, as shown in Figure 2. It is desirable to drive each link by a separate joint motor, as this would ease position control from a control logic viewpoint. The required joint torque to maintain the link in a certain position is merely the reaction moments at each joint. A two-link planar robot arm is schematically shown in Figure 3. Solving for the reaction moments, or required joint torque, results in the following relations

$$
\tau_{1}=\left(m_{1} g \frac{L_{1}}{2}+m_{2} g L_{1}+F_{L} L_{1}\right) \cos \left(\theta_{1}\right)+\left(m_{2} g \frac{L_{2}}{2}+F_{L} L_{2}\right) \cos \left(\theta_{1}+\theta_{2}\right)
$$




$$
\tau_{2}=\left(m_{2} g \frac{L_{2}}{2}+F_{L} L_{2}\right) \cos \left(\theta_{1}+\theta_{2}\right)
$$

In which,

$$
\begin{array}{ll}
\tau_{i}: & \text { The reaction moment at joint } i \\
\theta_{i}: & \text { The orientation of the robot arm } i \\
F_{L}: & \text { Weight of the load } \\
m_{L}: & \text { Mass of the load } \\
m_{i}: & \text { Mass of the robot arm } i .
\end{array}
$$

Equation (1) defines the reaction moment at the first joint, and equation (2) defines the reaction moment at the second joint. If $\theta_{1}$ is given, $\tau_{1}$ and $\tau_{2}$ have maximum and minimum values with respect to $\theta_{2}$ when $d \tau_{1} / d \theta_{2}, d \tau_{2} / d \theta_{2}$ are equal to zero.

$$
\frac{d \tau_{1}}{d \theta_{2}}=\frac{d \tau_{2}}{d \theta_{2}}=-\left(m_{2} g \frac{L_{2}}{2}+F_{L} L_{2}\right) \sin \left(\theta_{1}+\theta_{2}\right)=0
$$

We denote the maximum and minimum torques in the static simulation with $\tau_{s}^{\max }$, and $\tau_{s}^{\text {min }}$ respectively.

Figure 2 Multi-body dynamic simulation model in the 'closed-in' position and the 'manipulator extended' position (see online version for colours)

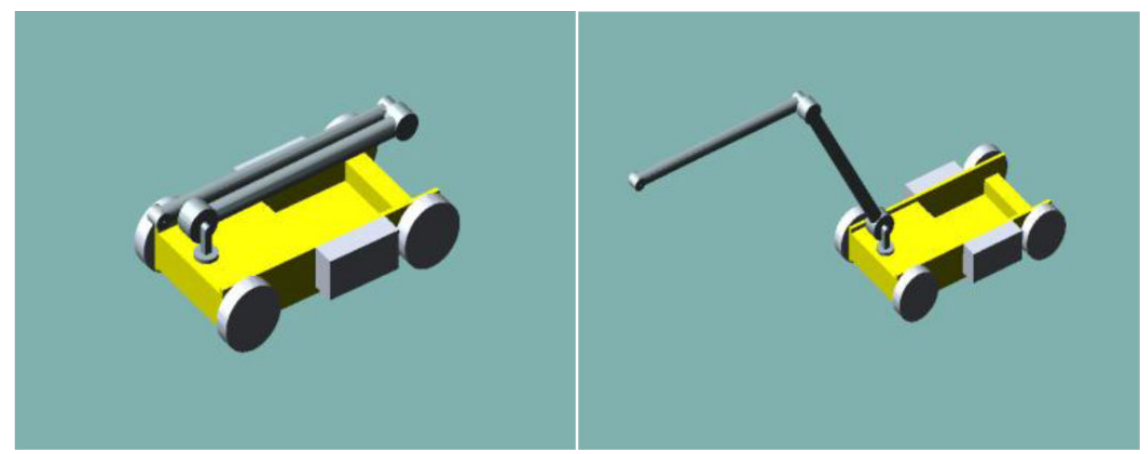

Figure 3 Schematic of a two-link robot arm

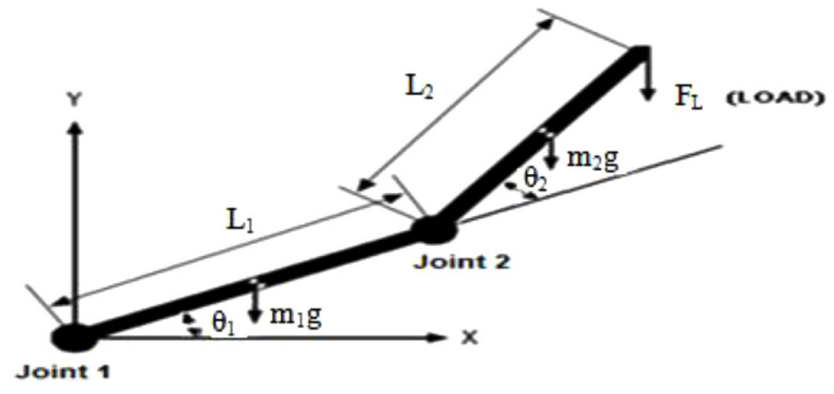


During the static simulation, the second joint angle, $\theta_{2}$, is varied from 0 to $2 \pi$, i.e., one full revolution, for each selected first joint angle. The joint torques are then calculated using the relations listed above. We can see from the equations that the maximum and minimum for both $\tau_{1}$ and $\tau_{2}$ are observed at the same orientation of the robot arm. By solving equation (3), we can conclude that at the worst-case orientations, the second joint angle is determined as $\theta_{2}=2 \pi-\theta_{1}$ if $0<\theta_{1}<\pi / 2$ and $\theta_{2}=\pi-\theta_{1}$ if $\pi / 2<\theta_{1}<\pi / 2$. Given the first link angle, the orientation of the second link, which results in maximum torque experienced by the robot actuators, is shown in Table 2.

Table 2 The link orientations that result in maximum torque experienced by the actuators

\begin{tabular}{lcc}
\hline First joint angle (Radian) & Second joint angle (Radian) & Manipulator orientation \\
\hline 0 & $0,2 \pi$ & $11 \pi / 6$ \\
$\pi / 6$ & $5 \pi / 3$ \\
$\pi / 3$ & $\pi / 2,3 \pi / 2$ \\
$\pi / 2$ & $\pi / 3$ & \\
$2 \pi / 3$ & $\pi / 6$ & \\
$5 \pi / 6$ & $0,2 \pi$ & \\
$\pi$ &
\end{tabular}

By setting the joint torque thresholds $T_{1}$ and $T_{2}$, the safe working range of the second joint can be determined under the given first joint angle range of $0<\theta_{1}<\pi / 2$.

$$
\begin{aligned}
& T_{1} \geq \tau_{1}, \quad T_{2} \geq \tau_{2} \\
& T_{1} \geq\left(m_{1} g \frac{L_{1}}{2}+m_{2} g L_{1}+F_{L} L_{1}\right) \cos \left(\theta_{1}\right)+\left(m_{2} g \frac{L_{2}}{2}+F_{L} L_{2}\right) \cos \left(\theta_{1}+\theta_{2}\right) \\
& \cos \left(\theta_{1}+\theta_{2}\right) \leq \frac{T_{1}-\left(m_{1} g \frac{L_{1}}{2}+m_{2} g L_{1}+F_{L} L_{1}\right) \cos \left(\theta_{1}\right)}{\left(m_{2} g \frac{L_{2}}{2}+F_{L} L_{2}\right)} \\
& T_{2} \geq\left(m_{2} g \frac{L_{2}}{2}+F_{L} L_{2}\right) \cos \left(\theta_{1}+\theta_{2}\right) \\
& \cos \left(\theta_{1}+\theta_{2}\right) \leq \frac{T_{2}}{\left(m_{2} g \frac{L_{2}}{2}+F_{L} L_{2}\right)} .
\end{aligned}
$$

From equations (6) and (8), we can determine that the first joint has a narrower safe working range than the second joint under the same joint torque threshold value, because the right hand side of equation (6) will always be smaller than that of equation (8). Similar analysis and conclusion can be obtained under the first joint angle range of $\pi / 2<\theta_{1}<\pi$. 
The static simulation model described above was evaluated under varying arm dimensions, masses, load size and joint angles. The model used in the static simulation has the dimensions $L_{1}=0.55 \mathrm{~m}, L_{2}=0.64 \mathrm{~m}, m_{1}=2.5 \mathrm{~kg}, m_{2}=2.5 \mathrm{~kg}, m_{L}=4 \mathrm{~kg}$, which are based on the measurements taken from an actual UGV manipulator. The same parameters are used in the dynamic simulation which will be presented next.

\subsubsection{Dynamic simulation framework}

Dynamic analysis generally provides more accurate results compared with the static study, since the former takes into account the arms inertia, interaction between various components, and other disturbances such as speed variations. However, the inclusion of these factors also increases the complexity of the dynamic model, which in turn imposes a challenge for the development of analytical solutions. Therefore, in most situations, a simulation approach has to be used to perform multi-body dynamic analysis with the consideration of these factors in the model.

In the multi-body dynamic simulation, all the components are modelled in a CAD system and converted into rigid bodies for use in MSC ADAMS, a multi-body dynamic simulation software. After all the parts are assembled, the complete model is exported into MSC ADAMS. The simulation calculates information such as lateral and longitudinal forces, torques, angular velocity and acceleration at each joint. The model also accounts for all centre of gravity locations in each component. Figure 2 shows the 3-D graphical rendering of the vehicle model in the 'closed-in' position and the 'manipulator extended' position.

During the dynamic simulation, for each selected first joint angle, $\theta_{1}$, the second joint angle, $\theta_{2}$, is varied from 0 to $2 \pi$ radian, i.e. one full revolution. This makes the manipulator move through a full range of motion and provides data for all operating states.

\subsubsection{Dynamic and static simulations results for joint torque saturation}

When the upper threshold of the joint torque for the link actuators is known, the failure of the robot manipulator will occur when the joint torque exceeds the threshold. For example, assuming that the joint torque threshold is $50 \mathrm{Nm}$, the first joint of the manipulator used in the simulation will fail in various positions. Various manipulator orientations are evaluated using both dynamic and static simulations. The results for dynamic simulation at two angular velocities, one with a typical operating speed of $10 \mathrm{deg} / \mathrm{sec}$ and the other with a much larger speed of $80 \mathrm{deg} / \mathrm{sec}$, along with the static simulation result at the first joint angle of $\pi / 6$, with a joint torque threshold of $50 \mathrm{Nm}$, is shown in Figure 4. The figure shows that speed and torque ripples create variations in the torque measurements in the dynamic simulation, as expected. Due to larger moment arms, speed variations create spikes that are more pronounced near the torque maxima in the dynamic simulation. Additionally, the maximum torque in the dynamic simulation does not increase significantly within typical operating speeds of the arm. In fact, using statistical hypothesis testing, we will show that a straightforward static simulation can provide a reasonable estimation for failure detection. In the next section, we will evaluate the validity of the conclusion under the selected operating speeds. 
Figure 4 Static and dynamic simulation torque profiles for the first robot joint with the first joint angle of $\pi / 6$ radians. (Top) operating speed of $10 \mathrm{deg} / \mathrm{sec}$. (Bottom) operating speed of $80 \mathrm{deg} / \mathrm{sec}$ (see online version for colours)
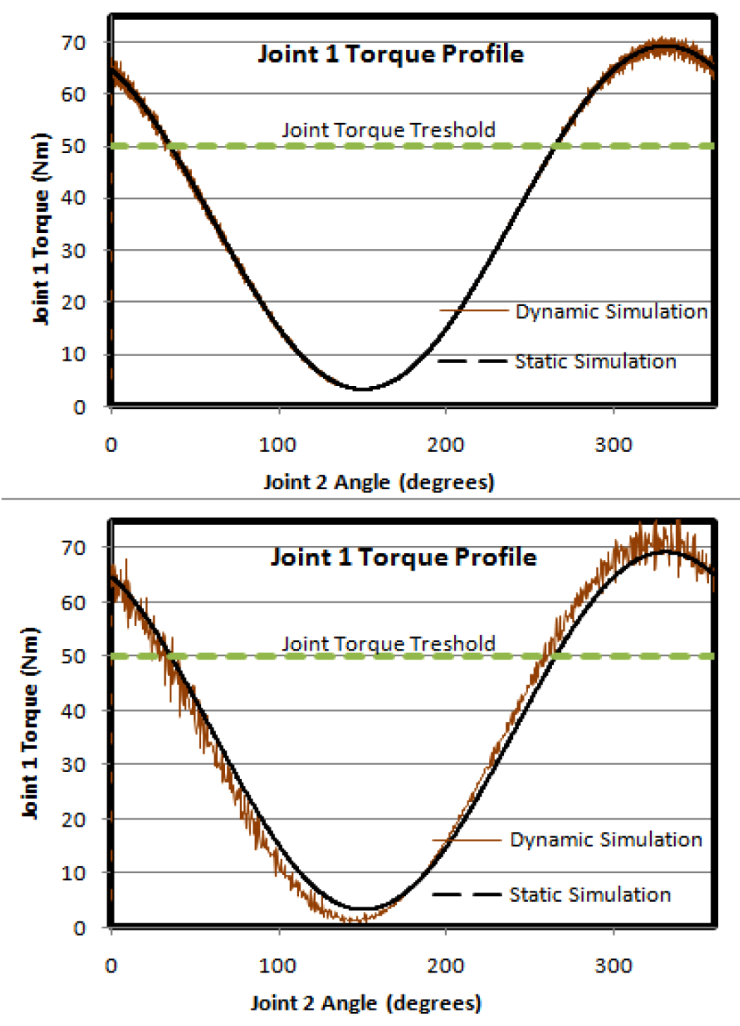

\subsubsection{Statistical hypothesis test for simulation comparison}

To further quantitatively assess the differences between static and dynamic simulation results and justify whether static simulation can be used instead of dynamic simulation for predicting failures, we will use statistical hypothesis tests. In this scenario, the failure occurs when $\tau_{d}^{\max }(\omega)$ exceeds the joint torque thresholds, where $\tau_{d}^{\max }(\omega)$ refers to the maximum torque obtained from the dynamic simulation when the arm is travelling at $\omega \mathrm{deg} / \mathrm{sec}$. Consequently, the comparison between static and dynamic simulation would be based on the accuracy of the static simulation in predicting $\tau_{d}^{\max }(\omega)$ within typical operating speeds. The advantage of static analysis is its simplicity and ease of use. On the other hand, the dynamic model provides more accurate and realistic results, but it is much more complex and time consuming to construct. As a result, when comparing the dynamic and static simulations, we would tolerate slight differences in their results by imposing a threshold for maximum allowable deviation of static results from their dynamic counterparts. This threshold is set based on the maximum deviation that is not of significance to the end user when studying a particular failure mode.

Since in this scenario the effect of speed variations are included as a part of dynamic simulation, the maximum torque during a full revolution of robot arm, which we will refer to as $\tau_{d}^{\max }(\omega)$, is a random variable whose variance is affected by the magnitude 
and frequency of the random speed ripples. If the maximum operating speed of the robot arm is $30 \mathrm{deg} / \mathrm{sec}$, the magnitude of the speed ripples are considered to be around $1 \%$ of this maximum operating speed.

A statistical hypothesis test will be used to verify whether deviation of the static simulation result from the dynamic simulation result is significant or not. A one-sided hypothesis test on the differences between the mean of the maximum torque measurements at each angular velocity with the calculated maximum torque in static simulation is constructed as follows

$$
\left\{\begin{array}{l}
H_{0}: \mu_{\tau_{d}^{\max }(\omega)}-\tau_{s}^{\max } \leq \Delta \\
H_{1}: \mu_{\tau_{d}^{\max }(\omega)}-\tau_{s}^{\max }>\Delta
\end{array}\right.
$$

In which $\Delta$ is the maximum allowable difference between the static and dynamic simulation results, and $\mu_{\tau_{\text {max }}(\omega)}$ is the mean of the maximum torque measurements in the dynamic simulation. With a prior belief that the static and dynamic simulations are both capable of predicting failures accurately, the rejection of null hypothesis in equation (9) is an indication that the static simulation is no longer capable of predicting the failure at a given allowable deviation level, and the dynamic simulation should be used instead.

The null hypothesis in equation (9) is rejected if:

$$
\frac{\bar{\tau}_{d}^{\max }(\omega)-\left(\tau_{s}^{\max }+\Delta\right)}{\frac{S_{\tau}}{\sqrt{n}}} \geq t_{\alpha, n-1} .
$$

In which $\bar{\tau}_{d}^{\max }(\omega)$ and $S_{\tau}$ are the sample mean and standard deviation of the maximum torque measurements. $\bar{\tau}_{d}^{\max }(\omega)$ and $S_{\tau}$ are calculated by running the dynamic simulation for $n$ complete revolutions, collecting the maximum torque value in each revolution, and computing the corresponding sample mean and standard deviation. Additionally, $t_{\alpha, n-1}$ is the $100(1-\alpha)$ th percentile of the $t$ distribution with $n-1$ degrees of freedom. Inequality (10) can be rewritten as:

$$
\bar{\tau}_{d}^{\max }(\omega) \geq \tau_{s}^{\max }+\Delta+t_{\alpha, n-1} \frac{S_{\tau}}{\sqrt{n}} .
$$

The right hand side of inequality equation (11) provides a rejection boundary for the hypothesis test. If $\bar{\tau}_{d}^{\max }(\omega)$ is larger than the rejection boundary, the hypothesis test is rejected, indicating that the deviation of static and dynamic simulations is significant and dynamic simulation should be used for failure analysis.

\subsubsection{Findings from the statistical hypothesis test}

The result from the static analysis agrees with the result from the dynamic analysis with a typical operating speed of $10 \mathrm{deg} / \mathrm{sec}$. Figure 5 depicts $\bar{\tau}_{d}^{\max }(\omega)$ at various angular velocities along with the hypothesis test rejection boundary obtained from equation (11). This figure shows that the static simulation model can be used for failure analysis, rather than a more complex dynamic simulation model, due to slow operating speeds of the UGV manipulator arms. However, static simulation is not always able to take the place of 
dynamic simulation because the joint torque increases as the robot operating speed increases due to inertial effects. With a typical maximum operating speed of about $30-50 \mathrm{deg} / \mathrm{sec}$, as shown in Figure 5, static simulation provides reasonable estimates for angular velocities as high as the maximum operating speed. However, the accuracy of estimates gradually deteriorates at higher angular velocities. The hypothesis test result verifies this observation by rejecting the static simulation results at angular velocities above $150 \%$ of the maximum operating speed.

Figure 5 Statistical comparison between dynamic and static simulations, for the first joint angle of $\pi / 6$ radians and $\Delta=\tau_{s}^{\max } \times 5 \%, n=10, \alpha=0.99$ (see online version for colours)

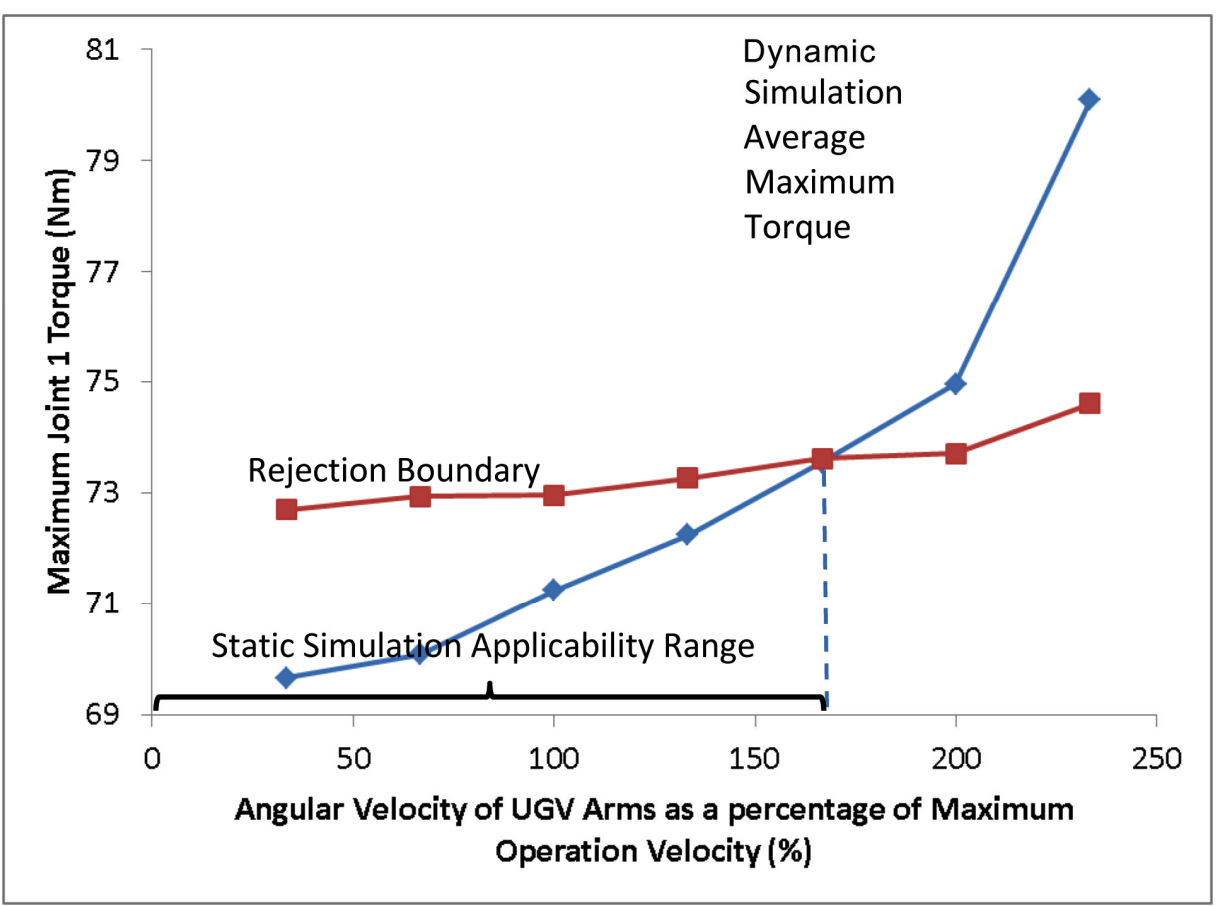

\subsubsection{Identifying the test range for the joint torque saturation failures}

In the previous section, it was shown that static simulation would provide reasonable approximation for torque saturation analysis within typical operating speeds. The developed static simulation model is further applied to identify the test range under various joint torque threshold values for joint torque saturation failures. Assuming the threshold values are 30,50 , and $70 \mathrm{Nm}$, the failure region versus the safe operating range of the manipulator, as shown in Figure 6. This plot will be very useful for acceptance testing planning, because it shows the boundaries of the operating range under different joint torque thresholds. For example, when the joint torque threshold of $50 \mathrm{Nm}$ is given, the circled line in Figure 6 forms a failure boundary. These failure ranges, which can potentially be costly during the actual test, should be avoided and excluded from the acceptance testing. The acceptance test scenario would then assess the UGV's capabilities in the safe operating regions. 
It should be noted that although both static and dynamic simulations have to be conducted for the purpose of comparison, the simulations and hypothesis tests do not need to be implemented and compared again when there are minor design changes, such as changes in the length of the robot arms. Additionally, for acceptance testing scenarios that share similar characteristics, both simulations do not need to be repeated, and the previously identified simulation scheme can be used. For instance, static simulation can be used for studying operations with UGV arms whose range of operating speeds are similar to those discussed earlier, without the need for constructing a dynamic simulation model. In addition, even if both simulation schemes are needed for a new acceptance testing scenario that does not share the characteristics of the previous simulated tests, the hypothesis test can largely reduce the analysis time by identifying conditions in which a static simulation can be used. In other words, instead of exploring the large space of dynamic parameters and environment by means of dynamic simulation, the hypothesis test result may suggest that analytical static simulations can be used to quickly identify the boundaries of safe operation.

Figure 6 Failure range and safe operating range of the two-link planar robot arm for threshold values $(30,50$, and $70 \mathrm{Nm})$ are shown in terms of first and second joint angle (see online version for colours)

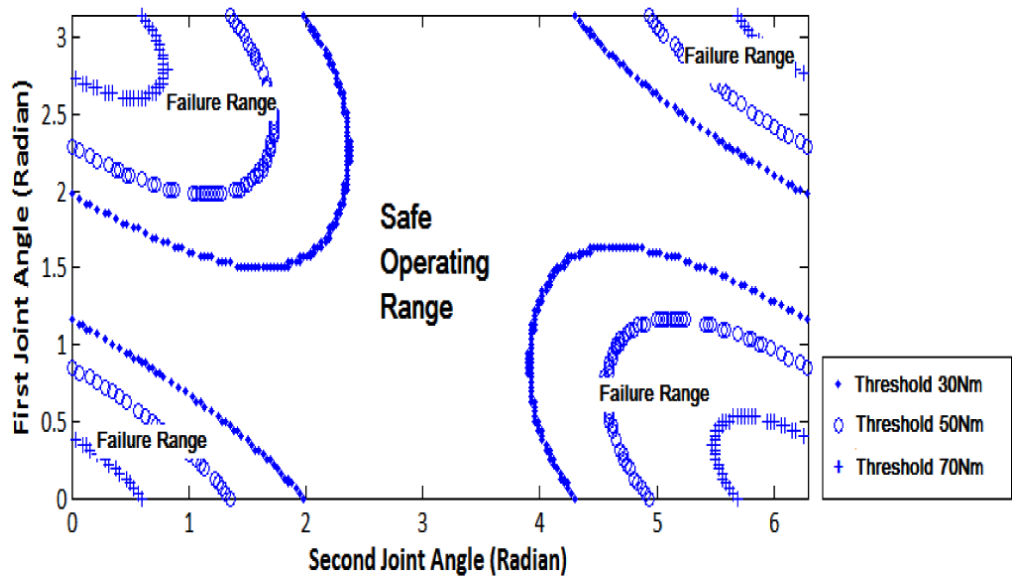

\subsection{Study of rollover failure}

\subsubsection{Identifying the test range for the rollover failures}

The static simulation model discussed in the previous section can be combined with rollover failure simulation. First, additional model parameters such as platform dimensions and weight are defined. Next, equations for static analysis are derived, and this static simulation model is implemented. Several initial robot orientations can be chosen to test whether the system rolls over while the robot arm travels through its full range of motion, and those robot orientations are shown in Figure 7. All these orientations are evaluated, and the result for the right-tilt orientation is shown in Figure 8. As shown, the initial robot orientation has a significant impact on failure and its safe operating range, and this result can provide guidance for operators to avoid rollover failures. Additional test methods can be based on these initial robot 
orientations, including a dragging capability test and degradation in lifting capability without flippers.

Figure 7 Example initial robot orientations for test set up. Test measures the safe operation range of the tilt angle for different robot arm orientations

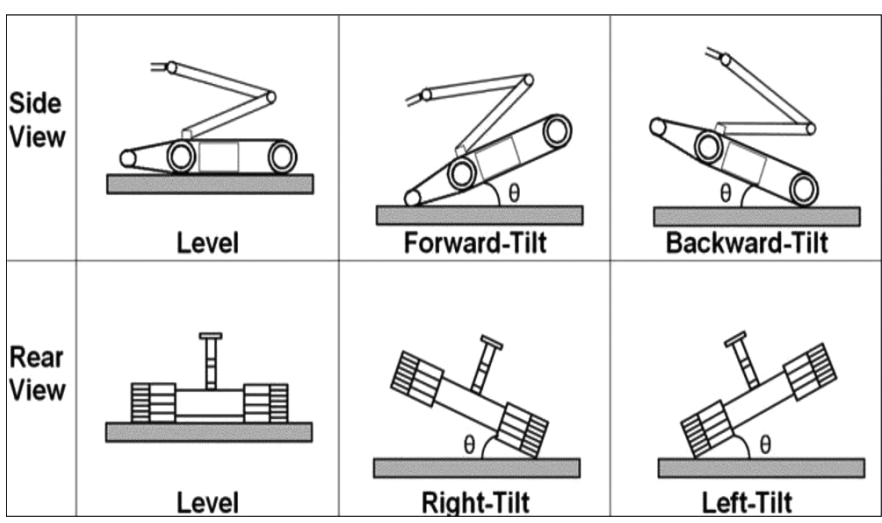

Figure 8 Failure range and safe operating range of the two-link planar robot arm for right-tilt (roll) angle of 30 and 40 are shown in terms of first and second joint angle (see online version for colours)

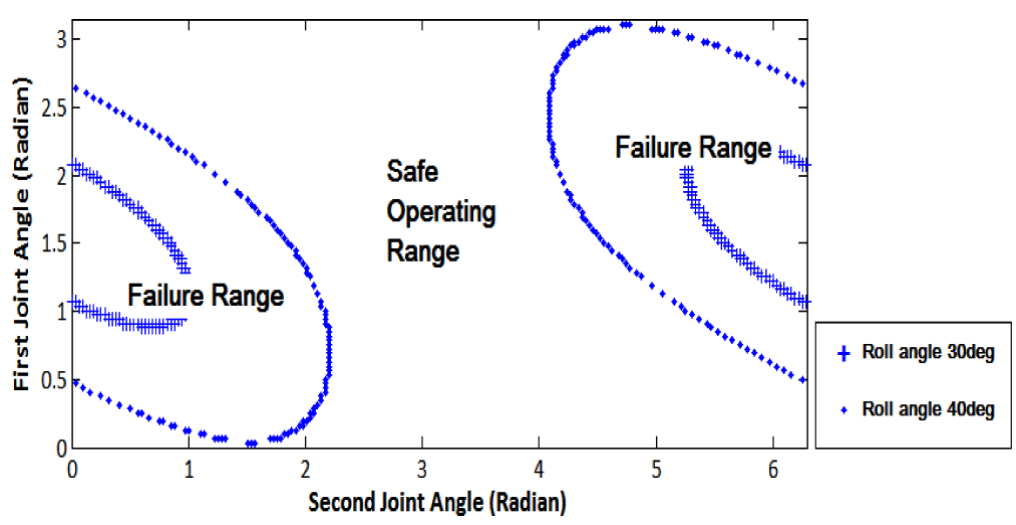

\section{Study of the suspension system and flip over failures}

\subsection{Scenario description}

UGVs are used in a variety of environmental conditions. As a result, it is essential to study the effect of diverse operational conditions, such as road bumpiness and roughness, on the mobility capabilities of the robot. In this scenario, the UGV moves across a bumpy road with mild roughness at various velocities. This scenario is inspired in part by the acceptance test methods for evaluating the mobility capabilities of emergency response robots conducted by NIST (Jacoff et al., 2009). Figure 9 shows the UGV on the road. A few interrelated failure modes can be studied under this setting as follows: 
1 When the forward velocity of the UGV is increased, as it drives on a rough road, the wheels intermittently lose contact with the road. The high inertia of the vehicle will raise the wheels, and can potentially lead to loss of vehicle contact with the road.

2 After the loss of wheel contact with the road, if the robot velocity is further increased, the UGV will experience excessive mechanical shocks. These shocks are introduced when the robot wheels come into contact with the road again after losing contact initially. They can result in failure of the suspension or damage electronic devices on the vehicle.

3 The UGV may also flip over when it moves at high velocities on a rough road surface. The loss of balance usually occurs when the UGV crosses parts of the road that have higher inclination angles.

The above failure modes are closely related. The relationships among them will be further elaborated in Section 5.6.

Figure 9 (Left) UGV crossing (left to right) a bumpy road. (Right) one-degree-of- freedom spring-damper suspension was added to the UGV model (see online version for colours)

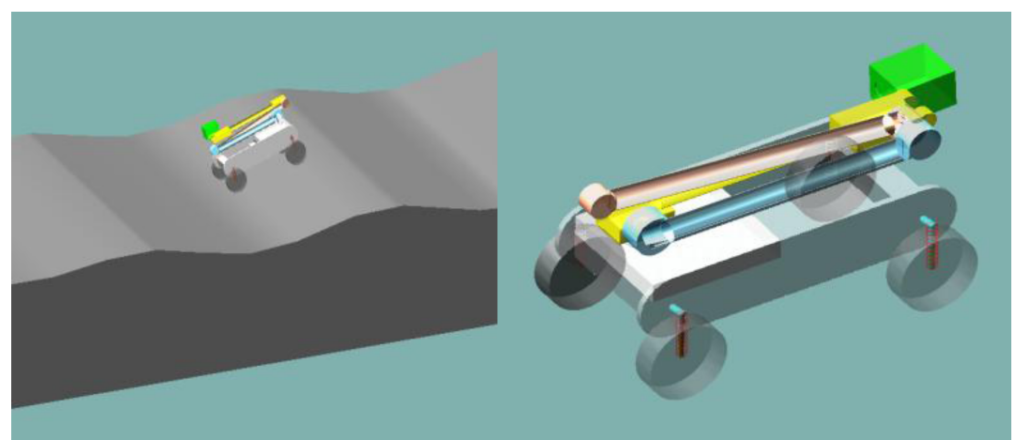

\subsection{Static simulation framework}

To conduct the static simulation, a 2-D representation of the UGV is considered. The corresponding free body diagram and the static equations are shown in Figure 10. The numerical values of parameters used in the equations are provided in Table 3. For the purpose of comparison between dynamic and static simulations, the maximum suspension force on a given road will be calculated. It is assumed that the wheels are locked and only the static friction between the wheels and the road prevents the UGV from sliding.

The central mass of the vehicle is closest to the rear left wheel. Consequently, the rear left suspension experiences the largest forces due to the asymmetrical centre of mass of the UGV. Since mechanical shocks are associated with large reaction forces in the suspension, special care should be directed to the rear left wheel for failure analysis of the suspension system.

Figure 11 shows that the orientation of the UGV on the road has a significant effect on the suspension system's loads. In particular, the maximum suspension load occurs when the UGV is positioned on parts of the road with largest angles. In addition, road roughness is too small to change the orientation of the vehicle and consequently its effect on the static simulation is negligible. The maximum angle of the UGV on the road 
depends on the size of the UGV, amplitude, and spatial frequency of the bumps on the road. The negative angles refer to down-slopes, and positive angles indicate upward slopes. Figure 11 illustrates that $F_{1}$ increases with larger up-slopes (larger positive $\theta$ ), while $F_{2}$ increases with larger down-slopes (larger negative $\theta$ ).

Table 3 Parameters used in the static and dynamic simulations

\begin{tabular}{lc}
\hline Parameter name & Value \\
\hline$L_{1}$ & $21.2 \mathrm{~cm}$ \\
$L_{2}$ & $28.7 \mathrm{~cm}$ \\
$L_{3}$ & $23 \mathrm{~cm}$ \\
UGV mass in static analysis & $18.47 \mathrm{~kg}$ \\
Wheel's mass & $0.166 \mathrm{~kg}$ \\
Coefficient of static friction & 0.8 \\
Coefficient of dynamic friction & 0.7 \\
Spring constant & $5000 \mathrm{~N} / \mathrm{m}$ \\
Damping coefficient & $250 \mathrm{~N}-\mathrm{sec} / \mathrm{m}$ \\
UGV typical arm operating speed & $10 \mathrm{deg} / \mathrm{sec}$ \\
UGV maximum operating speed & $6 \mathrm{miles} / \mathrm{h}$ \\
$G_{e}$ & 0.01 \\
$G_{s}$ & 20 \\
$G_{a}$ & $1 \times 10^{-6}$ \\
\hline
\end{tabular}

Figure 10 Free body diagram of the UGV
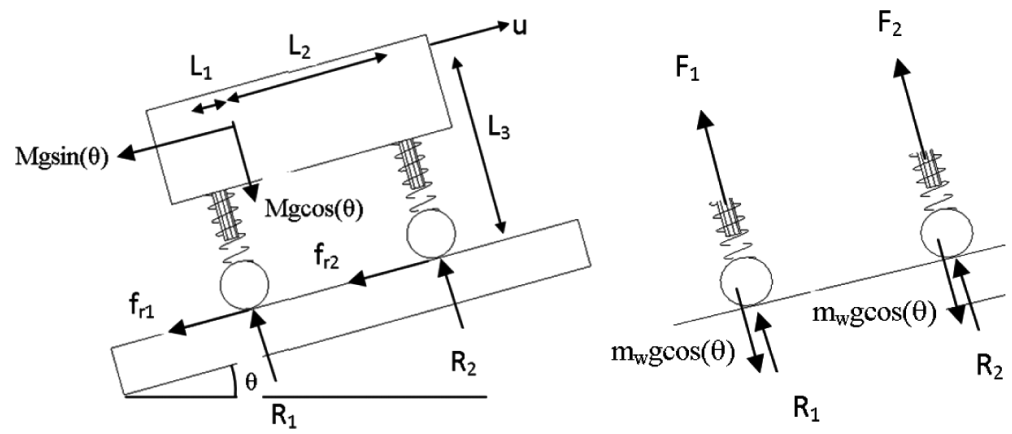

$$
\begin{aligned}
& R_{1}=\frac{M g\left(L_{2} \cos (\theta)+L_{3} \sin (\theta)\right)}{\left(L_{1}+L_{2}\right)} \\
& R_{2}=\frac{M g\left(L_{1} \cos (\theta)-L_{3} \sin (\theta)\right)}{\left(L_{1}+L_{2}\right)} \\
& F_{1}=R_{1}-\left(m_{w} g\right) \cos (\theta) \\
& F_{2}=R_{2}-\left(m_{w} g\right) \cos (\theta) .
\end{aligned}
$$


In which,

$R_{i}$ : $\quad$ Reaction force of the wheel $i$

$F_{i}: \quad$ Suspension force associated with the wheel $i$

$F_{r i}: \quad$ Static friction between the wheels and the road

$\theta: \quad$ Orientation/angle of the UGV on the road

$m_{w}: \quad$ Mass of the wheel

M: $\quad$ UGV total mass including wheels

Figure 11 The static simulation results for the rear and front suspension force (see online version for colours)

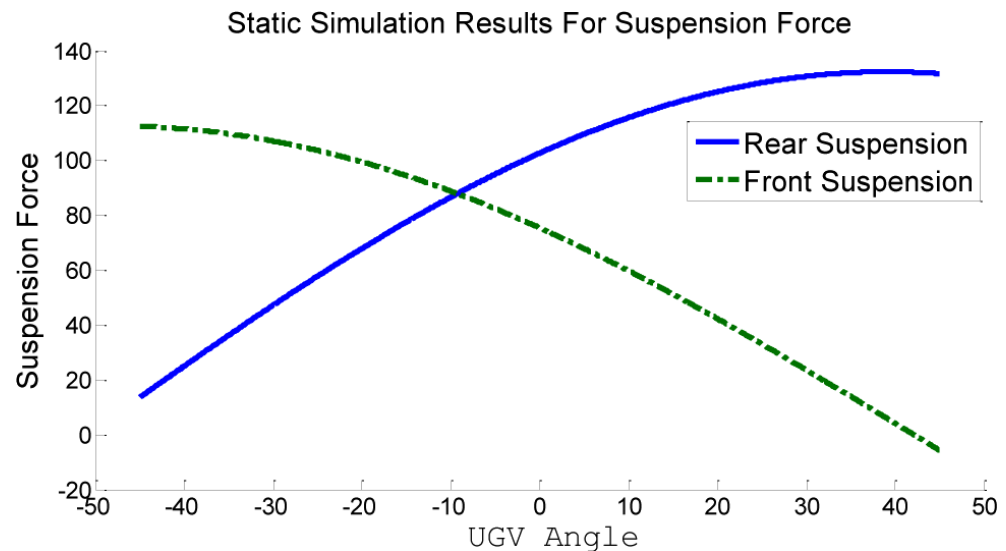

\subsection{Dynamic simulation framework}

Similar to the torque saturation scenario in Section 4, all the components such as road profiles were modelled in a CAD system and converted into rigid bodies for use in MSC ADAMS. The parameters used in dynamic simulation, such as coefficients of friction between the road and the UGV wheels, range of operating speeds, and suspension system details are included in Table 3. The road profile is assumed to follow a sine function with amplitude of $0.075 \mathrm{~m}$. The simulation considers a variety of spatial frequencies for the road. Additionally, the road model includes mild roughness. The roughness of the road was generated using MSC ADAMS/CAR road generation toolbox. To summarise, the road profile follows the expression below:

$$
Y(x)=0.075 \times \sin \left(\omega_{r} x\right)+\varphi\left(G_{e}, G_{s}, G_{a}, x\right)
$$

in which $\omega_{r}$ is the spatial frequency of the road, and $G_{e}, G_{s}, G_{a}$ are white noise elevation, slope and acceleration parameters respectively. These parameters are used for generating the road roughness according to Sayers (1988). Using the values in Table 3, our road profile represents a bumpy Portland-cement concrete road with a rigid to smooth-rigid roughness characteristic. Additionally, larger $\omega_{r}$ will decrease the distance between bumps, which results in sharper road angles and higher excitation frequencies. 
To capture the effect of mechanical shocks and road roughness, a one-degree-offreedom suspension (spring-damper) system was added to the model. Figure 9 depicts the 3-D graphical rendering of the model. The properties of the suspension system, such as spring and damping coefficients are provided in Table 3. These parameters were selected to provide a combination of low natural frequency and large enough stiffness and damping to prevent excessive spring deformation. Spring deformation was critical due to the small size of the UGV and limitations on suspension stroke. In addition, considering the road profiles and typical operating speeds of the UGV, the excitation of the suspension system induced by movement of the UGV on the road will be smaller than the natural frequency of the suspension system.

The road roughness in the dynamic simulation results in random suspension forces. The effect of road roughness on the suspension loads is depicted in Figure 12. As shown, the maximum force differs slightly from one road cycle to the other. As also shown in the figure, the road consists of several bumps, and the road cycles are equality spaced, including one bump each.

Figure 12 The effect of road roughness on force profiles. Comparison of rear suspension forces on a rough vs. a smooth bumpy road (see online version for colours)

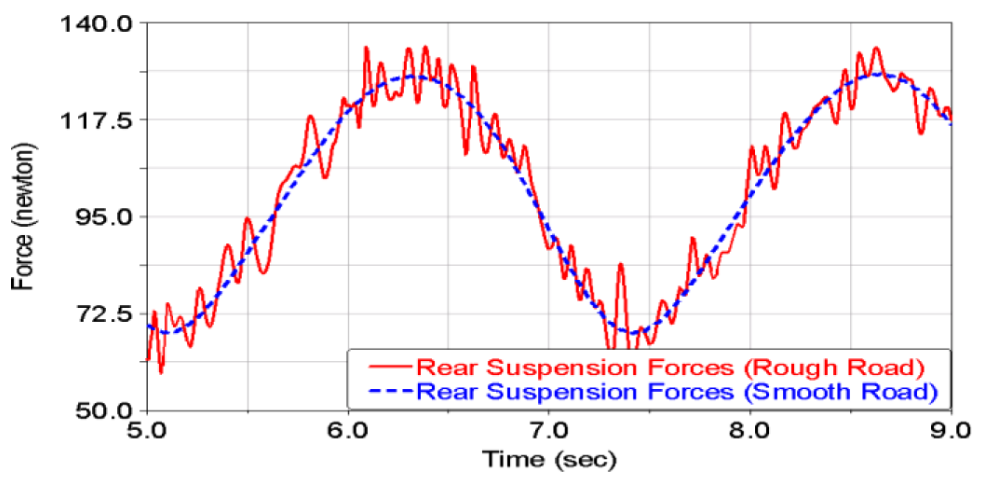

\subsection{Statistical hypothesis test for simulation comparison}

Similar to the torque saturation scenario, the goal is to assess the static simulation effectiveness for predicting failure. The failure mode under consideration is the suspension system breakdown, which is caused by excessive mechanical shocks. Therefore, the measure of interest in the comparisons is the maximum force that the suspension experiences on the road. The deviation of static results from the dynamic simulation outcome is determined via statistical tests. A one-sided hypothesis test on the differences between the mean of the maximum suspension forces in the dynamic simulation at different velocities with the calculated maximum force in the static simulation is constructed as follows:

$$
\left\{\begin{array}{l}
H_{0}: \mu_{F_{d}^{\max }(V)}-F_{s}^{\max } \leq \Delta \\
H_{1}: \mu_{F_{d}^{\max }(V)}-F_{s}^{\max }>\Delta
\end{array} .\right.
$$

in which $F_{s}^{\max }$ is the maximum suspension force on a given road obtained from the static simulation, and $\mu_{F_{d}^{\max }(V)}$ is the mean of the maximum force measurements in the dynamic 
simulation when the robot goes through one complete road cycle with a forward velocity $V$.

The null hypothesis in equation (17) is rejected if:

$$
\frac{\bar{F}_{d}^{\max }(V)-\left(F_{s}^{\max }+\Delta\right)}{\frac{S_{F}}{\sqrt{n}}} \geq t_{\alpha, n-1} .
$$

in which $\bar{F}_{d}^{\max }(V)$ and $S_{F}$ are the sample mean and standard deviation of the maximum force measurements. $\bar{F}_{d}^{\max }(V)$ and $S_{F}$ are calculated by running the dynamic simulation for $n$ road cycles, collecting the maximum force value in each cycle, and computing the corresponding sample mean and standard deviation. Inequality equation (18) can be rewritten as:

$$
\bar{F}_{d}^{\max }(V) \geq F_{s}^{\max }+\Delta+t_{\alpha, n-1} \frac{S_{F}}{\sqrt{n}} .
$$

The right hand side of inequality equation (19) provides a rejection boundary for the hypothesis.

\subsection{Findings from the statistical hypothesis test}

Static analysis fails to predict failure within typical operating speeds. Figure 13 depicts $F_{d}^{\max }(V)$ along with the hypothesis test rejection boundary obtained from equation (19). The value of $\Delta$ is assumed to be $5 \%$ of the $F_{s}^{\max }$ in Figure 13. The statistical test shows that only for a small range of operating speeds, about a quarter of the maximum operating speed, the static simulation can provide a reasonable estimate for the maximum force in the suspension system. Additionally, this figure shows that this conclusion holds for any reasonable value of $\Delta$ selected by the user, since $\bar{F}_{d}^{\max }(V)$ increases rapidly at higher velocities. Typical operating speed range for the UGV is between 0 and 6 miles/hour.

Figure 13 Statistical test for comparison of dynamic and static simulations for $\omega_{r}=4$,

$\Delta=F_{s}^{\max }=5 \%, n=10, \alpha=0.99$ (see online version for colours)

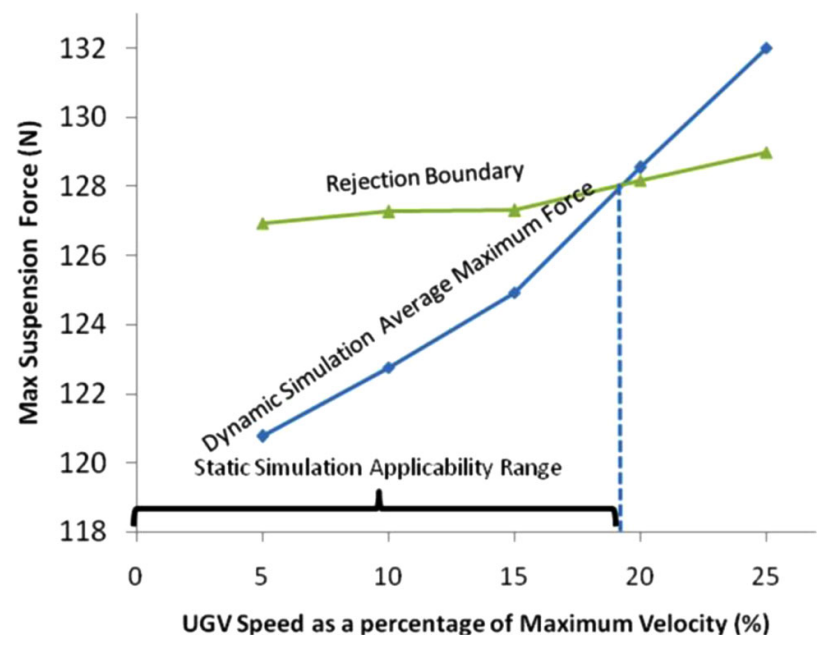




\subsection{Statistical assessment of safe operating speed using dynamic simulation}

In the previous section, it was shown that static simulation fails to provide accurate results. Unlike the torque saturation scenario, the means of dynamic forces in a bumpy road setting quickly diverged from their static counterparts within typical operating speeds. Consequently, we use dynamic simulation to develop boundaries of safe operating speed.

As mentioned earlier, three failure modes associated with this scenario are intermittent loss of contact between the wheels and the road, excessive mechanical shocks, and finally flip over. Intermittent contact is an early indication of more severe failures such as mechanical shocks. Therefore, this event can provide suitable insight for failure prevention. Flip over, on the other hand, occurs at higher operating speeds, while severe mechanical shocks mainly take place at velocities close to the onset of flip over. Figure 14 shows the relation among the three events. This graph also provides a general guideline for how the UGV should be operated in various road conditions. Since intermittent loss of contact does not always result in an actual failure, (but rather is an early indication of other failures), to develop boundaries of safe operating speeds, we will focus on the failure mode associated with excessive mechanical shocks. This failure is directly correlated with the maximum force that the UGV suspension experiences on a road. The corresponding hypothesis test for failure analysis is as follows:

$$
\left\{\begin{array}{l}
H_{0}: \mu_{F_{d}^{\max }(V)} \leq F_{\text {failure threshold }} \\
H_{1}: \mu_{F_{d}^{\max }(V)}>F_{\text {failure threshold }}
\end{array} .\right.
$$

in which, $F_{\text {failure threshold }}$ is the failure threshold above which the suspension failure takes place. The hypothesis test is rejected if:

$$
\frac{\bar{F}_{d}^{\max }(V)-F_{\text {failure threshold }}}{\frac{S_{F}}{\sqrt{n}}} \geq t_{\alpha, n-1} .
$$

Figure 14 Loss of contact is an early indication of suspension system and flip over failures based on dynamic simulation (see online version for colours)

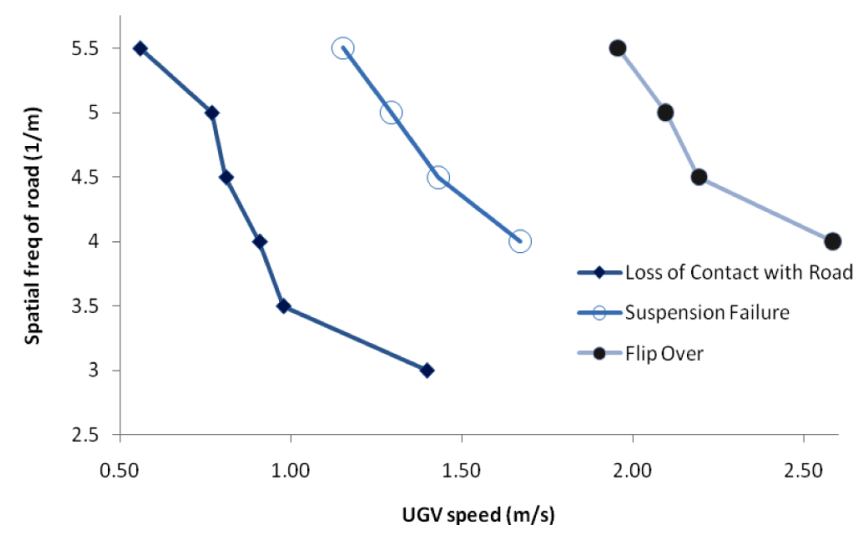


We can rewrite inequality equation (21) to obtain a rejection boundary for the hypothesis test as follows:

$$
\bar{F}_{d}^{\max }(V) \geq F_{\text {failure threshold }}+\frac{S_{F}}{\sqrt{n}} t_{\alpha, n-1} .
$$

Figure 15 shows the $\bar{F}_{d}^{\max }(V)$ measurements along with the corresponding rejection boundary from equation (22). In this figure, it is assumed that failure occurs when the robot experiences forces in excess of $400 N$, i.e., $F_{\text {failure threshold }}$, which is more than three times as large as $\bar{F}_{d}^{\max }(V)$ at low velocities. Given the current failure limit, the allowable operating speed range on this bumpy road is about $62 \%$ of the maximum operating velocity. As a result, the failure range will include any velocities above this speed threshold. This failure range, which can result in costly damages to the UGV components, can then be excluded from acceptance testing immediately. A similar type of analysis can be easily applied to roads with different surface roughness, bump size and road profiles.

Figure 15 Boundaries of safe operation for suspension breakdown and flip over prevention on a typical bumpy road with mild roughness for $\omega_{r}=4$ based on dynamic simulation (see online version for colours)

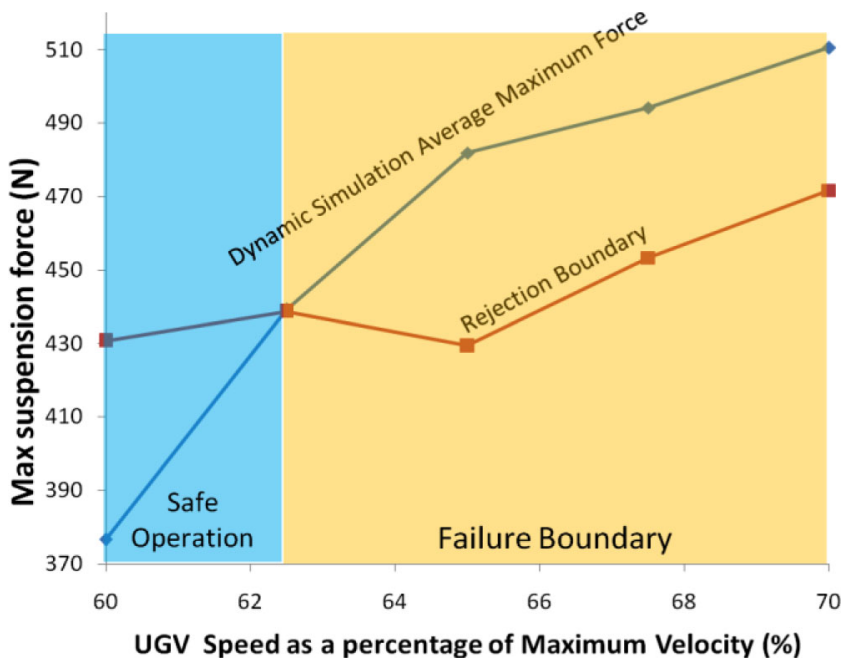

To summarise, we showed that dynamic simulation results provide general guidelines to how the UGV should be operated on various rough bumpy roads, and can significantly help with failure prevention and design of more effective physical acceptance testing scenarios.

\section{Conclusions and future work}

In this paper, we introduced a simulation-based acceptance testing methodology that can be used as a complementary tool for physical testing under a number of typical operational failures, which include joint torque saturation, rollover, flip over, and 
suspension system breakdown. To capture operational variations and environmental uncertainties, a statistical hypothesis test is proposed to quantitatively test whether the difference between dynamic simulations and static simulations significantly affect the decision of UGV operational states. The results of statistical hypothesis testing show that in the analysis of joint torque saturation and rollover failures that arise under slow operational speeds, decisions on the failure states are consistent between the static and dynamic simulations. Therefore, static simulations can be used as a good approximation of dynamic simulations in these situations. In contrast, in the case of analysing the flip over and suspension failures, dynamic simulations cannot be approximated by static simulations. Moreover, we further show how to develop the boundaries of safe operation based on the simulation results. These results can help provide a guideline in the design of efficient physical testing scenarios, which not only help avoid unnecessary testing procedures, but also can avoid some scenarios leading to severe damages to the UGVs during the physical tests.

MSC ADAMS, which was used to compare the static and dynamic simulation results in the paper, is a widely used dynamic simulation tool in the industry. Recently, Pepper et al. (2007) performed physical validation tests for the Talon Robot's simulation models. They investigated the similarities and differences between the simulation results from the physical tests for improving simulation models and their parameters under the specific scenarios, such as passing through steps and stairs, or directed perception and grasping dexterity tests. However, there are no physical tests conducted for torque saturation and flip-over failures that are presented in this paper. Thus, future work will be needed to experimentally validate those dynamic simulation models using physical facilities such as those available at NIST (Messina and Jacoff, 2006), and the US Army Aberdeen Proving Ground (Tricomo, 2009).

\section{Acknowledgement}

UNCLASSIFIED: Dist. A. Approved for public release. This research was supported in part by the Ground Robotics Reliability Center (GRRC) at the University of Michigan, with funding from government contract DoD-DoA W56H2V-04-2-0001 through the United States Army Tank Automotive Research, Development and Engineering Center. The authors also wish to thank Mr. Jongsoo Choi for his assistance with the simulations.

\section{References}

Carlson, J. (2004) Analysis of How Mobile Robots Fail in the Field, Masters Thesis, Department of Computer Science and Engineering, University of South Florida, Tampa, FL.

Carlson, J. and Murphy, R. (2003) 'Reliability analysis of mobile robot', Paper presented at the IEEE International Conference on Robotics and Automation (ICRA), Taipei, Taiwan, Vol. 1, pp.274-281.

Carlson, J. and Murphy, R. (2005) 'How UGVs physically fail in the field', IEEE Transactions on Robotics, Vol. 21, No. 3, pp.423-437.

Carlson, J., Murphy, R. and Nelson, A. (2004) 'Follow-up analysis of mobile robot failures', Paper presented at The IEEE International Conference on Robotics and Automation (ICRA), New Orleans, LA, pp.4987-4994. 
Godler, I., Ohnishi, K. and Yamashita, T. (1994) 'Repetitive control to reduce speed ripple caused by strain wave gearing', Paper presented at The Industrial Electronics, Control and Instrumentation, 1994. IECON '94., 20th International Conference on, Bologna, Italy, Vol. 2, pp.1034-1038.

Guonian, Y., Haiying, H., Hao, S., Junhu, W. and Guoji, S. (2010) 'The whole vehicle system dynamics modeling and simulation for transportation vehicle', Paper presented at The Mechanic Automation and Control Engineering (MACE) International Conference on, Wuhan, China, pp.698-701.

Jacoff, A., Messina, E., Huang, H., Virts, A. and Downs, A. (2009) Standard Test Methods for Response Robots (pamphlet), ASTM International committee on homeland security applications; Operational equipment; Robots (E54.08.01). Version 2009.88.

Kramer, J. and Murphy, R. (2006) 'Endurance testing for safety, security and rescue robots', Performance Metrics for Intelligent Systems Workshop, NIST Special Publication, pp.247-254.

Lam, B., Panda, S. and Xu, J-X. (2000) 'Reduction of periodic speed ripples in PM synchronous motors using iterative learning control', Paper presented at The Industrial Electronics Society, 2000. IECON 2000. 26th Annual Conference of the IEEE, Nagoya, Aichi, Japan, Vol. 2, pp.1406-1411.

Messina, E. and Jacoff, A. (2006) 'Performance standards for urban search and rescue robots', Proceedings of the SPIE Defense and Security Symposium, Orlando, FL, Vol. 6230, DOI: $10.1117 / 12.663320$.

Micire, M. (2002) Analysis of the Robotic-Assisted Search and Rescue Response to the World Trade Center Disaster, Master Thesis, Department of Computer Science and Engineering, University of South Florida, Tampa, FL.

Norris, G. (1995) 'Boeing's seventh wonder', Spectrum, IEEE, Vol. 32, No. 10, October, pp.20-23.

Pepper, C., Balakirsky, S. and Scrapper, C. (2007) 'Robot simulation physics validation', Proceedings of the Performance Metrics for Intelligent Systems PERMIS, Gaithersburg, MD, USA, pp.97-104.

Purdy, E. (2007) Ground Robotics Technology, Presentation Slide, Joint Ground Robotics Enterprise, Department of Defence, URL: http://www.jointrobotics.com/Jan07/ Tab\%20D\%20-\%20Purdy.pdf, Accessed 30 October 2011.

Ren, Z-B., Yu, Z-A. and Liang, J-W. (2009) 'Study and implementation on high accuracy servo system for robot joint', Paper Presented at The Control and Decision Conference (CCDC '09), Guilin, China, pp.1673-1676.

Sayers, M. (1988) Dynamic Terrain Inputs to Predict Structural Integrity of Ground Vehicles, UMTRI Report, UMTRI-88-16.

Tilbury, D. and Ulsoy A.G. (2011) 'A new breed of robots that drive themselves', ASME Mechanical Engineering Magazine, Vol. 133, February, pp.28-33.

Tricomo, S. (2009) Army AL\&T Online, April Retrieved 11 May, 2011, from http://www. usaasc.info/alt_online. 